\title{
SIMULAÇÃO COMPUTACIONAL DO RESFRIAMENTO DA MANGA TOMMY ATKINS
}

\author{
ROCHA, Lucas A. de B. ${ }^{1}$ \\ SILVA, José de Castro ${ }^{1}$ \\ ANINGER, Lucas $^{1}$ \\ CASTRO, José de ${ }^{2}$ \\ FIGUEIREDO NETO, Acácio $^{3}$
}

Recebido em: 2016.01.21

Aprovado em: 2016.09 .14

ISSUE DOI: $10.3738 / 1982.2278 .1615$

RESUMO: Este trabalho tem como objetivo simular o processo de resfriamento da manga Tommy Atkins utilizando software ANSYS Mechanical. Os resultados evidenciam o potencial do uso da simulação computacional aplicada à agricultura como opção na predição do comportamento termofísico de produtos hortícolas. O método, de baixo custo computacional, mostrou-se apropriado para a caracterização do processo de resfriamento, tendo como provável benefício a aplicabilidade no estudo do processo de resfriamento de outros produtos agrícolas

Palavras-chave: Simulação numérica. Resfriamento com ar forçado. Frutas.

SUMMARY: This paper simulates the process to refrigerate mango (Tommy Atkins) using ANSYS Mechanical software. The results put in evidence the benefits of computer simulation applied into the agriculture field predicting the thermo-physic behavior of vegetable products. The low cost method applied in this study seemed applicable for the characterization of the mango refrigeration process, also being a possible method in the study of other agriculture products.

\section{Keywords:}

\section{INTRODUÇÃO}

Segundo os dados do Anuário Brasileiro de Fruticultura, Santos (2014), o Brasil encontra-se em terceiro lugar no ranking mundial de produtores de frutas, tendo apenas China e Índia à sua frente. Dentre as 25 espécies de frutas que o Brasil produz e exporta, a manga possui uma posição de destaque, posicionando o país como $7^{\circ}$ maior exportador da fruta no mundo (FAO, 2013).

Mesmo sendo o Estado de São Paulo a região com maior área de pordução de manga, a Região Nordeste apresenta os sistemas de cultivo mais técnificados, especialmente na região do Vale do Submédio do São Francisco (Silva et al., 2009), sendo a responsável por cerca de $80 \%$ da exportação de mangas no Brasil (LIMA, 2013).

Após a colheita, a vida da manga é limitada pela deterioração fisiológica, devido ao amadurecimento da fruta, desenvolvimento de patógenos e perda de água, causando enrugamento e murchamento da frutaprejudicando assim, o valor comercial. Cerca de 50\% das perdas por ano de produtos hortícolas frescos se deve à falta de do tratamento pós-colheita adequado. Como a diminuição da temperatura até os níveis recomendados ara cada produto é o procedimento mais adequado para aumentar ávida útil dos produtos, o uso da Cadeia do Frio (CF) se torna indispensável. (TERUEL et al., 2003).

Dentre os sistemas que compõem a CF, o resfriamento com sistemas de ar forçado, ocupam uma posição de destaque, pelas vantagens que oferecem.

Nestes sistemas o produto é acondicionado no interior de uma câmara refrigerada, que além do

\footnotetext{
${ }^{1}$ Estudante de Eng. Mecânica da UNIVASF

${ }^{2}$ Docente de Eng. Mecânica da UNIVASF

${ }^{3}$ Docente de Eng. Agrícola da UNIVASF
} 
sistema convencional de refrigeração, ventiladores são acoplados para atuarem como exaustores. As embalagens utilizadas para acondicionar os produtos devem contemplar área de aberturas suficientes para facilitar a troca térmica entre o produto e o ar resfriado. Além de que o arranjo de produtos dentro da mesma, deve favorecer o maior contato possível com o ar, o que intensifica a perda de calor, diminuindo significativamente o tempo de resfriamento dos produtos.

A literatura reporta resultados de estudos de simulação do processo de resfriamento de produtos hortícolas, alguns exemplos são o limão (AMENDOLA, 2003), grãos de milho (Rocha, 2012), figo (AMENDOLA, DUSSÁN-SARRIA; RABELLO, 2009) e morango (PIROZZI E;MENDOLA, 2005). Os resultados destes trabalhos destacam a utilidade de desenvolver estudos de simulação do resfriamento, buscando minimizar o tempo necessário para obter respostas que auxiliem a tomada de decisão, como por exemplo, a modificação do projeto de embalagens, a distribuição do produto na câmera e na embalagem, assim como modificações no sistema de distribuição do ar. Ao mesmo tempo, a taxa de resfriamento é dependente do tamanho, forma e propriedades térmicas do produto (DEFRAEYE et al., 2013).

A adoção das técnicas de simulação computacional, como o CFD (Computational Fluid Dynamics), vem se tornando uma ferramenta alternativa que auxilia e influencia na diminuição dos custosa relativos á experimentação e o redução do tempo necessário para se ter respostas.

Com o passar dos anos, a versatilidade, a facilidade e precisão do uso oferecido pela DFC levou a sua aceitação abrangente dentro da comunidade de engenharia agrícola, tornando-se evidente em vários trabalhos da área (Silva et al., 2014 Bartzanas et al. (2013), Lee et al. (2013), Piscia et al. (2015), Wu (2013) e Ambaw et al. (2013).

O objetivo desse estudo foi simular as condições de resfriamento forçado de mangas da variedade Tommy Atkins através de simulação computacional utilizando um software comercial (ANSYS® Workbench $\left.{ }^{\mathrm{TM}} 15.0\right)$.

\section{MATERIAL E MÉTODOS}

Os estudos foram realizados no Departamento de Engenharia Mecânica da Universidade Federal do Vale do São Francisco (UNIVASF). O software ANSYS® utilizado para realizar a simulação foi escolhido devido à sua disponibilidade no laboratório de DFC (Dinâmica de Fluidos Computacional) da UNIVASF - Campus Juazeiro.

O procedimento computacional está fromado por sete etapas:

- Definição do sistema de análise a ser utilizado (Analysis System);

-Seleção do material no banco de dados;

- Modelagem da geometria pela ferramenta nativa do ANSYS ${ }$, o DesignModeler ${ }^{\mathrm{TM}}$;

- Geração da malha e consequentemente, o seu refino (Model);

- Aplicação da configuração do problema e inserção das condições de contorno (Setup);

- Solução das equações inerentes ao problema (Solution);

- Apresentação dos resultados (Results).

Dentre os diversos sistemas de análise disponíveis na suíte de ferramentas do ANSYS® Workbench ${ }^{\mathrm{TM}}$, o que melhor supre as necessidades para solução da simulação é o TransientThermal, o qual é uma pré-configuração da ferramenta $A N S Y S^{\circledR}$ para análises de transferência de calor em regime transiente.

Para que o modelo computacional tenha comportamento semelhante ao da fruta é necessário inserir as propriedades do material lembrando que Para os cálculos nas equações de transferência de calor, 
as propriedades termofisicas do produto em estudo são necessárias. As propriedades termofisicas da manga. Estas propriedades (Tabela 1) foram determinadas experimentalmente por alguns pesquisadores, após experimentos seguindo métodos definidos na literatura Simões, 1997; Mattos, 2007.

Tabela 1. Propriedade Termofísicas da Manga Tommy Atkins.

\begin{tabular}{ll}
\hline Propriedade & Valor \\
\hline Densidade & $1070 \mathrm{kgm}^{-3}$ \\
Calor Específico & $3,95 \mathrm{kJkg}^{-1} \cdot{ }^{\circ} \mathrm{C}^{-1}$ \\
Condutividade Térmica & $0,58 \mathrm{Wm}^{-1} \cdot \mathrm{C}^{-1}$ \\
\hline
\end{tabular}

A modelagem da geometria foi realizada pelo programa SolidWorks ${ }^{\circledR}$. Como referência para o modelo virtual 3D, foram utilizadas fotos do fruto, tiradas durante o experimento em laboratório. Tendo essas imagens como base, desenhou-se no programa um semielipsoide semelhante à manga cortada, usada no experimental. A Figura 1 apresenta o modelo e suas dimensões $(0,1 \mathrm{~mm}$ e $0,09 \mathrm{~mm})$.

Figura 1. Dimensões do modelo virtual (raios do elipsoide).

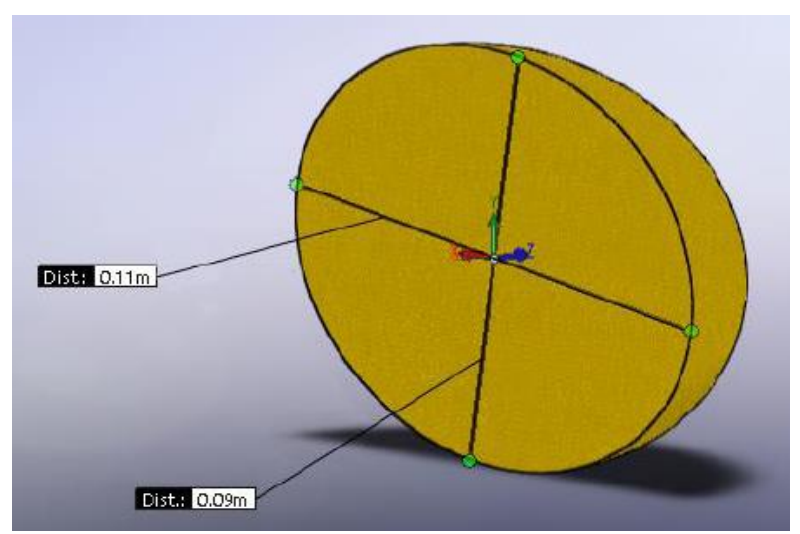

Foi gerada uma malha composta por elementos mistos (hexaédricos e tetraédricos), totalizando 23841 elementos. A malha foi incialmente gerada automaticamente pelo ANSYS® e, em seguida, foi realizado um refino para que o domínio fosse melhor dividido, tendo mais pontos, e com isso, um resultado melhor. A malha refinada utilizada pode ser vista na Figura 2.

Figura 2. Vista superior da malha.

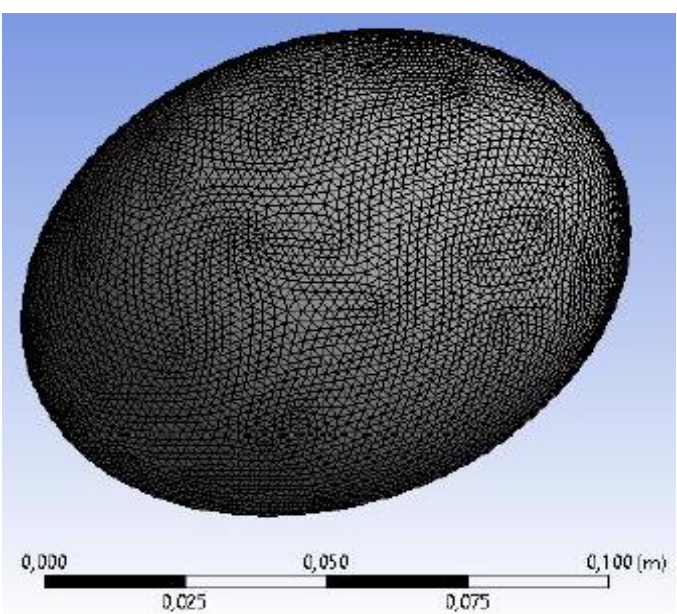


Para a análise do tipo transiente, o método de solução iterativo aplicado foi o Método QuaseNewton, o qual precisa apenas que o gradiente da função objetivo esteja disponível em cada iteração, para que, desta forma, ao calcular as variações no gradiente a cada iteração, seja construído um modelo para a função objetivo suficiente para gerar uma convergência superlinear (ANDRETTA, 2010).

Já o solver das equações utilizado foi o Método dos Gradientes Conjugados otimizado com o uso da técnica de pré-condicionamento de Decomposição Incompleta de Cholesky (IC). Este solver foi selecionado por ser versátil, e de fácil implementação, tanto em ambiente sequencial quanto em paralelo.

O solver gera um mapa da distribuição de todas as variáveis pelo domínio. Esse resultado deve ser processado para que possa ser facilmente visualizado e analisado. Este é o principal objetivo da etapa de pós-processamento, a qual é essencial para uma avaliação compreensiva da simulação (SMALE et al., 2006).

\section{RESULTADOS E DISCUSSÃO}

Com os valores pretendidos, temperatura de ponto de equilíbrio e o tempo que leva para a fruta chegar neste ponto, a simulação recebe o estado final que deve ser obtido, isso é, todas as condições de contorno necessárias, apresentadas na Tabela 2.

Tabela 2. Condições de contorno da simulação.

\begin{tabular}{cc}
\hline Condição de Contorno & Valor \\
\hline Temperatura Ambiente & $-8,5^{\circ} \mathrm{C}$ \\
Temperatura Inicial do Fruto & $13{ }^{\circ} \mathrm{C}(\mathrm{T} 1) / 23,1{ }^{\circ} \mathrm{C}(\mathrm{T} 2)$ \\
Temperatura Final do Fruto & $0{ }^{\circ} \mathrm{C}$ \\
Tempo & $57 \min (\mathrm{T} 1) / 63 \mathrm{~min}(\mathrm{~T} 2)$ \\
\hline
\end{tabular}

Após as simulações, o coeficiente convectivo foi estimado e ajustado, um coeficiente convectivo constante que representasse de forma aceitável as variações do mesmo ao longo do tempo e incluindo a influência da velocidade do ar. Assim como Castro e Amendola (1999), Teruel (2000) e Amendola e Teruel (2005) fizeram, estimaram o valor de h, ajustando os dados encontrados numericamente aos dados obtidos via experimento prático.

Foram obtidos então, dois valores de coeficiente convectivo, um admitindo a temperatura inicial do fruto igual a $23^{\circ} \mathrm{C}$, e outro admitindo a temperatura inicial igual a $13^{\circ} \mathrm{C}$, sendo estes coeficientes respectivamente, 160,5 e $82,35 \mathrm{Wm}^{-2} \cdot{ }^{\circ} \mathrm{C}^{-1}$. Nota-se que o coeficiente convectivo utilizando a temperatura maior é aproximadamente o dobro da menor, devido ao fato de que na primeira simulação em $\mathrm{t}=0$ a temperatura é $10^{\circ} \mathrm{C}$ maior do que na segunda, e apenas 6 minutos de disparidade no tempo de homogeneização, o que implica em um grande aumento no coeficiente.

O uso deste método possibilitou a obtenção da distribuição das temperaturas internas ao longo do tempo, como pode ser visto na Figura 3. Sendo que, de forma experimental, a obtenção da mesma distribuição seria muito mais complexa (TERUEL et al., 2001). 
Figura 3. Distribuição das temperaturas internas

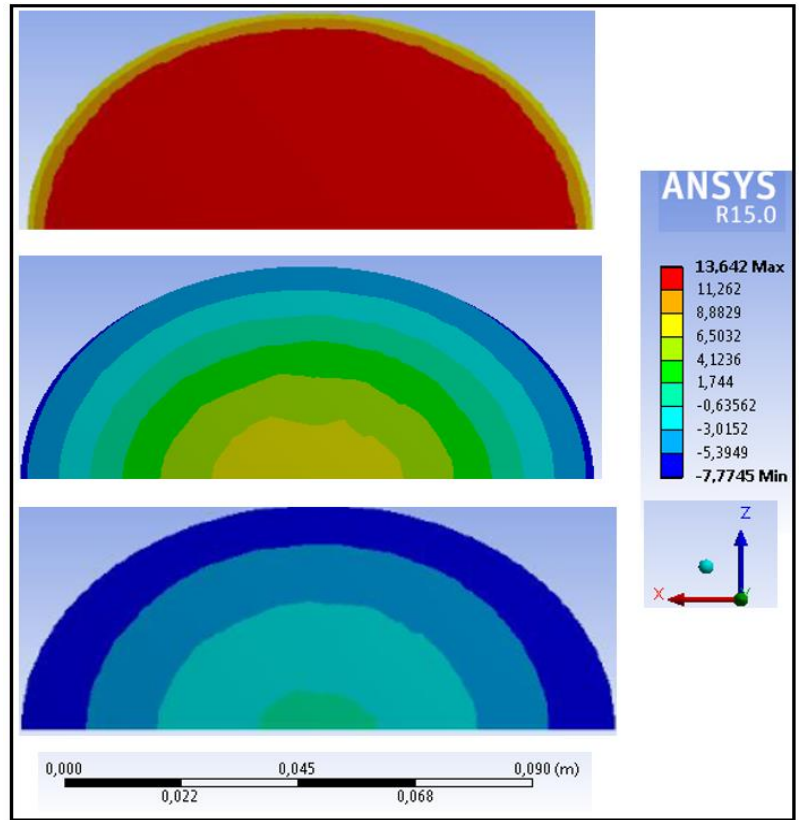

Para definir a melhor malha a ser utilizada, um teste de convergência de malha foi realizado com diferentes configurações Inicialmente se utilizou a malha automática gerada pelo próprio software, com 461 elementos; em seguida definiu-se o método de construção de elementos como Método Misto, o qual gera elementos hexaédricos e tetraédricos no modelo, criando uma malha com 7356 elementos.

Após o refino realizado foi definindo o tamanho do maior elemento presente, neste caso $7 \mathrm{~mm}$, gerando assim uma malha com 23481 elementos. Por último o tamanho máximo de elemento foi reduzido para $5 \mathrm{~mm}$, causando um tempo de processamento duas vezes maior do que o da definição anterior, e gerou uma malha com 45947 elementos.

Ao analisar o comportamento da temperatura (Figura 4), percebe-se que a terceira malha, de elementos de $7 \mathrm{~mm}$, se aproxima da de $5 \mathrm{~mm}$ e possui um tempo de processamento bem mais reduzido.

Figura 4 - Teste de Malha.

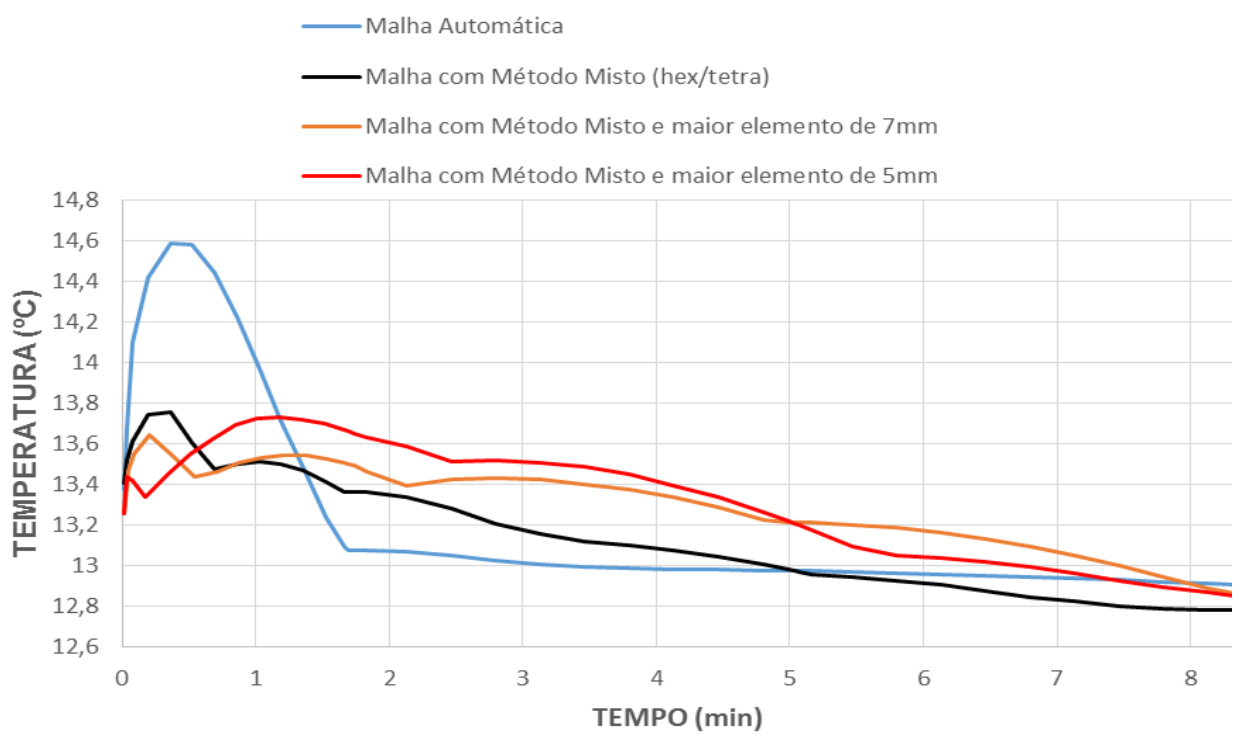




\section{CONCLUSÕES}

O método provou-se apropriado para a caracterização do processo de resfriamento, tendo como benefícios a aplicabilidade para outros produtos agrícolas e pequeno custo computacional.

A simulação numérica é um instrumento apropriado para o progresso do conhecimento deste procedimento, mas a suscetibilidade do coeficiente convectivo às diversas condições e parâmetros demanda o prosseguimento deste tipo de estudo.

\section{REFERÊNCIAS}

AMBAW, A. et al. 2013. The use of CFD to characterize and design post-harvest storage facilities: Past, present and future. Computers and Electronics in Agriculture, 93, 184-194.

AMENDOLA, M. (2002). Simulação numérica do processo de resfriamento rápido por ar forçado de limão. In: Congresso Nacional de Matemática Aplicada e Computacional 25, 2003. São José do Rio Preto: Anais... São José do Rio Preto: SBMAC, 2003a. p.143.

AMENDOLA, M., TERUEL, B.J. (2005). Esquema implícito e uso de splines para a simulação numérica do processo de resfriamento de frutas esféricas. Revista Brasileira de Engenharia Agrícola e Ambiental, 9, 1, 78-82.

AMENDOLA, M., DUSSAN-SARRIA, S., RABELLO, A.A. (2009). Determinação do coeficiente convectivo de transferência de calor de figos submetidos ao resfriamento rápido. Revista Brasileira de Engenharia Agrícola e Ambiental, 3, 2, 176-182.

ANDRETTA, M. (2010). Métodos Quase-Newtons. São Carlos: Universidade de São Paulo. 30 slides: color. Slides gerados a partir do software Power Point. Disponível em:

<http://www.icmc.usp.br/ andretta/ensino/aulas/sme5720-2-11/quasenewton.pdf> Acesso em: 02 de Junho de 2015.

Ansys Mechanical APDL Thermal Analysis Guide, Realease 15.0, 2015.

BARTZANAS, T. et al. 2013. Computational fluid dynamics applications to improve crop production systems. Computers and Electronics in Agriculture, 93, 151-167.

CASTRO, L.R., AMENDOLA, M. (1999). Simulação numérica do processo de transferência de calor em vegetais esféricos. In: CONGRESSO IBERO-LATINO-AMERICANO EM MÉTODOS

COMPUTACIONAIS E ENGENHARIA, 20, 1999, São Paulo. Anais... São Paulo. 1 CD ROM.

DEFRAEYE, T. et al. (2013) Forced-convective cooling of citrus fruit: Package design. Journal of Food Engineering. 118, 8-18

FOOD AND AGRICULTURE ORGANIZATION - FAO. FAOSTAT. 2013. Disponível em: <http://faostat.fao.org>. Acesso em: 18 dez. 2014.

FORTUNA, A.O. (2000). Técnicas Computacionais para Dinâmica dos Fluidos: Conceitos Básicos e Aplicações. São Paulo: Editora da Universidade de São Paulo.

LEE, I.-B. et al. 2013. The past, present and future of CFD for agro-environmental applications.

Computers and Electronics in Agriculture, 93, 168-183. 
LIMA, J.R.F. (2013). Exportações de manga produzida no Submédio do Vale do São Francisco no Período de 2003 - 2012. Disponível em:

<http://www.infoteca.cnptia.embrapa.br/bitstream/doc/956079/1/COT154.pdf〉. Acesso em: 10 jan. 2015.

MATTOS, J.S. (2007). Bando de dados de propriedades termofísicas de produtos hortícolas. Dissertação (Mestrado em Engenharia Agrícola), Universidade Estadual de Campinas.

NORTON, T, SUN, D. (2006). Computational fluid dynamics (CFD) - an effective and efficient design and analysis tool for the food industry: A review. Trends in Food Science \& Technology. City - State, $17,600-620$.

PFAFFENBACH, L.B.et al.. (2003). Efeito da Atmosfera Modificada e da Refrigeração na Conservação Pós-Colheita de Manga Espada Vermelha. Revista Brasileira de Fruticultura. 25, 3, 410-413.

PIROZZI, D.C.Z, AMENDOLA, M. (2005). Modelagem matemática e simulação numérica do resfriamento rápido de morango com ar forçado. Engenharia Agrícola, 25, 1, 222-230.

PISCIA, D. et al. 2015. A method of coupling CFD and energy balance simulations to study humidity control in unheated greenhouses. Computers and Electronics in Agriculture, 115, 129-141

ROCHA, K.S.O. (2012). Modelagem e simulação do processo de resfriamento de grãos armazenados em silos graneleiros por meio das leis de conservação de energia e massa. Tese (Doutorado em Engenharia Agrícola), Universidade Federal de Viçosa.

SILVA, J. de C. (2014). Simulation of airflow in cold chambers using the openfoam ${ }^{\circledR}$ computational fluid dynamics (CFD) software. African Journal of Agricultural Research. 9, 1, 1-7.

SILVA, P.C.G. (2009). Dinâmica e crise da fruticultura irrigada no Vale do São Francisco. In: Gomes da SILVA, A.; CAVALCANTI, J. S. B.; WANDERLEY, M. de N. B. (orgs). Diversificação dos espaços rurais e dinâmicas territoriais no Nordeste do Brasil. João Pessoa: Ed. Zarinha Centro de Cultura, p. 69-95.

SIMÕES, M.R. (1997). Propriedades Termofísicas de Polpa de Manga. Dissertação (Mestrado em Engenharia de Alimentos), Universidade Estadual de Campinas.

SMALE, N.J. et al. (2006). A review of numerical models of airflow in refrigerated food applications. International Journal of Refrigeration. 29, 911-930.

SANTOS, C.E. et al., 2014. Anuário Brasileiro da Fruticultura. Editora Gazeta Santa Cruz, Santa Cruz do Sul.

TERUEL, M.B.J. (2000). Estudo teórico experimental do resfriamento de laranja e banana com ar forçado. Tese (Doutorado em Engenharia Mecânica), Universidade Estadual de Campinas.

TERUEL, B.et al.. (2001). Estudo teórico do resfriamento com ar forçado de frutas de geometrias diferentes. Ciência e Tecnologia de Alimentos, 21, 2, 228-235.

TERUEL, B., CORTEZ, L., NEVES FILHO, L. (2003). Estudo Comparativo do Resfriamento de Laranja Valência com Ar Forçado e com Água. Revista Ciência e Tecnologia de Alimentos, 23, 2, 174-178.

WU, B. (2013). Advances in the use of CFD to characterize, design and optimize bioenergy systems. Computers and Electronics in Agriculture, 93, 195-208. 
